\title{
SUPERCONDUCTING TRAVELLING WAVE RING WITH HIGH GRADIENT ACCELERATING SECTION
}

\author{
P. Avrakhov, Euclid TechLabs, LLC, Rockville, MD, 20850
}

\author{
N. Solyak, Fermi National Accelerator Laboratory, Batavia, Ill., 60510
}

\begin{abstract}
Use of a superconducting travelling wave accelerating (STWA) structure [1] instead of a standing wave cavity has major advantages in increasing the accelerating gradient in the ILC. In contrast with standing wave cavity STWA requires feedback loop, which sends wave from the structure output to input, making a superconducting travelling wave ring (STWR). One or few input couplers need to excite STWR and compensate power dissipations due to beam loading. To control travelling wave regime in the structure two independent knobs can be used for tuning both resonant ring frequency and backward wave. We discuss two variants of the STWR with one and two feed couplers.
\end{abstract}

\section{INTRODUCTION}

The principal goal of this work is to study travelling wave superconducting (SC) accelerator concepts to increase the accelerating gradient of a SC structure and, therefore to reduce the length (and hence the cost) of the accelerator. A superconducting travelling wave accelerator (STWA) allows the increase in the accelerating gradient of a superconducting structure larger by a factor of $1.2 \div 1.4$ over than that of the TESLA and alternative designs with a new shape: low-loss or reentrant [2].

As described in the February 7, 2007 ILC Reference Design Report (RDR) [2], the accelerating gradient is to be about $31.5 \mathrm{MeV} / \mathrm{m}$, the c.m. energy $500 \mathrm{GeV}$, and the ILC collider length $31 \mathrm{~km}$. To reach the required energy of $500 \mathrm{GeV}$ the accelerating system should have the length of $22 \mathrm{~km}$ and includes of about 16,000 one-meter long 9-cell superconducting cavities. Groups of 8 or 9 such cavities would be installed in a cryomodule. Any improvements in cavity performance will have big impact to the cost and efficiency of the ILC project. Two new proposed cavity designs: low-loss and re-entrant cavities aims to increase accelerating gradient or the gradient acceptance margin.

As show in the Table 1, the travelling wave accelerating structure has the best performances with respect to the surface magnetic and electric fields and the field uniformity along the accelerating structure. For the same gradient STWA has of $\sim 24 \%$ lower magnetic fields compare with TESLA type cavity. TW structure has much lower sensitivity to the frequency errors of the individual cells. In the current ILC designs the length of SW structure is limited to $1 \mathrm{~m}$, mostly because of field flatness requirements. As a result, there is an unavoidable space (gap) between $1 \mathrm{~m}$ long structures of about $280 \mathrm{~mm}$ that reduces the effective gradient by about $22 \%$. The TW structure has no such fundamental limitation and the length of STWA structure may be up to the length of cryomodule $(10 \mathrm{~m})$ if technology of the SC cavity fabrication and surface processing allows it. This means that the effective accelerating gradient can be increased up $22 \%$, giving an overall $46 \%$ gain over the ILC SW structure. Presented STWA structure has a $60 \mathrm{~mm}$ aperture and phase advance per cell of $105^{\circ}$, which was chosen for the technological reasons.

Table 1: Comparison between TESLA, Low-Loss, ReEntrant and STWA structures (1 meter)

\begin{tabular}{|c|c|c|c|c|}
\hline Cavity Parameters & TTF & LL60 & RE60 & STWA $\left(105^{\circ}\right)$ \\
\hline Aperture $[\mathrm{mm}]$ & 70 & 60 & 60 & 60 \\
\hline Coupling, $k_{c c} \quad[\%]$ & 1.9 & 1.52 & 1.57 & 3.35 \\
\hline$E_{\text {peac }} / E_{\text {acc }}$ & 2.0 & 2.36 & 2.28 & 1.94 \\
\hline$H_{\text {pead }} / E_{\text {acc }}[\mathrm{mT} / \mathrm{MV} / \mathrm{m}]$ & 4.15 & 3.61 & 3.54 & 3.05 \\
\hline$R_{s h} / Q \quad[\Omega]$ & 1036 & 1206 & 1140 & 1808 \\
\hline$G \cdot R_{s h} / Q \quad\left[\Omega^{2}\right]$ & $2.7 \cdot 10^{5}$ & $3.1 \cdot 10^{5}$ & $3.3 \cdot 10^{5}$ & $3.21 \cdot \cdot 10^{5 *}$ \\
\hline
\end{tabular}

$\left(^{*}\right)$ includes extra $9.2 \%$ waveguide losses.

\section{BASIC CONCEPTS}

Application of a superconducting accelerating structure with nonzero group velocity requires recuperation of the RF energy, i.e. the transmitted wave has to be returned to the section without any reflection from the input and output ports. Appearance of the reflected wave in the accelerating section increases the total surface electric and magnetic fields at the same accelerating gradient and accordingly reduces the KE and KH ratios. In 1949 the original idea of resonant ring with "warm" TW accelerating section was proposed by R.-Shersby-Harvie and Mullett [3]. The superconducting travelling-wave accelerator with feedback was considered in series of SLAC publications in 1968-1971 [4, 5], where the advantages of the TW accelerating scheme with feedback over the conventional SW SC systems were discussed.

Let us consider a mechanism of travelling wave excitation in the superconducting resonant ring with TW accelerating section. The resonant ring can be fed by one, two or more RF couplers depending on the accelerating section length and acceptable power level of the couplers. We will examine in detail one- and two-couplers feed schemes. It should be noted that the well known feed scheme with one directional coupler doesn't work in this case, because under the power multiplication factor of the SC TW resonator $\sim 10^{4}$, the requirements for the coupler directivity and inner ring reflection become impracticable. 
Both suggested schemes use non directional (standard TTF-III) couplers. Those methods of travelling wave excitation are successfully used for RF sources with circular beams deflecting in a rotating RF field - gyrocons and magnicons $[6,7]$.

The first scheme uses two input couplers that excite independently both partial standing waves comprising the resulting traveling wave. Each input coupler supplies half of the total power. The phases of the partial modes are shifted by of about $\pi / 2$. In addition, the first scheme (see Fig. 1) includes the structure, the feedback couplers, the feedback waveguide, and a special matching element ("matcher") that compensates reflections caused the input couplers, system imperfections, tuning errors, etc.

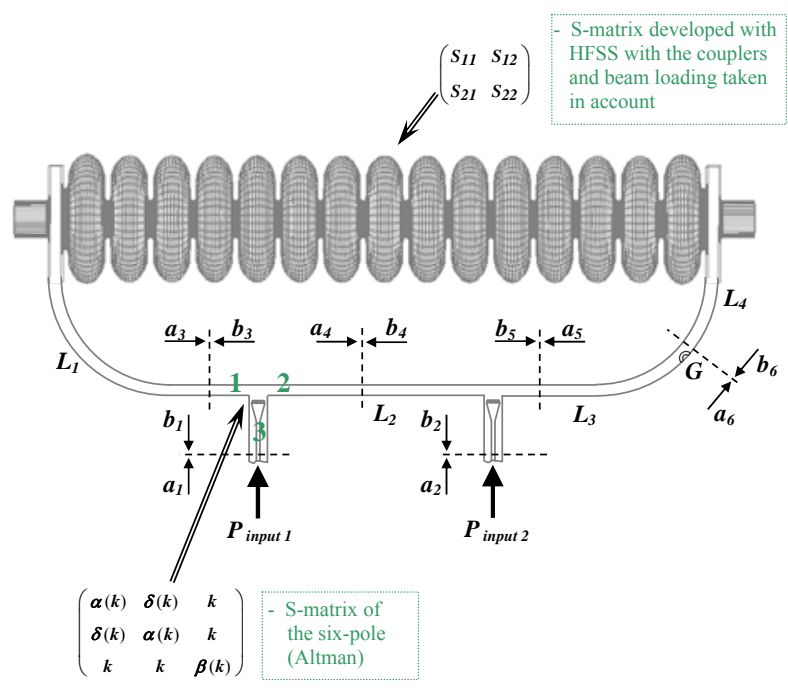

Figure 1. Two-coupler model of the resonant travelling wave ring with STWA cavity.

The following notation is used:

$L_{1}, L_{2}, L_{3}, L_{4}$ - the length of the waveguide sections between the resonance ring elements

$G$ - matcher reflection coefficient;

$\alpha(k)$ - reflection from the shoulders 1 and 2 of the T-joint; $\beta(k)$ - reflection from the shoulder 3;

$\delta(k)$ - transmission from the shoulder 1 to shoulder 2 ;

$k$-transmission from the shoulder 3 into 1 and 2 of the Tjoint .

The scattering matrix formalism is used for the system analysis. Each element is characterized by its own scattering matrix that depends on the element properties and its location in the system as shown in Fig. 1. The Smatrix of the structure and the feedback coupler is calculated numerically. The beam loading is taken into account. The input coupler is described by six-pole matrix (see, for example, ref. [8]).

The proposed model can be fully described by a system of equations with the right-hand element at the any point along the frequency scale. The bandwidth is rather narrow, of $\sim 10^{-6}$ for these types of devices ( $\mathrm{T}$-joint and matcher) and the S-matrix elements do not depend on the frequency. The feedback waveguide are $160 \mathrm{~mm}$ wide and exhibit normal dispersion. S-matrix of the resonance $\operatorname{ring} M(f)$ is equal:

\begin{tabular}{|c|c|c|c|c|c|c|c|}
\hline-1 & $S_{11} \cdot e^{-2 i \varphi_{1}}$ & 0 & 0 & 0 & 0 & $S_{21} \cdot e^{-2 i\left(\varphi_{1}+\Phi_{3}\right)}$ & 0 \\
\hline$\alpha_{1}$ & -1 & 0 & $\delta_{1} \cdot e^{-0.5 i \varphi_{2}}$ & 0 & 0 & 0 & 0 \\
\hline$\delta_{1} \cdot e^{-0.5 i \varphi_{2}}$ & 0 & -1 & $\alpha_{1} \cdot e^{-i \varphi_{2}}$ & 0 & 0 & 0 & 0 \\
\hline 0 & 0 & $\alpha_{2} \cdot e^{-i \varphi_{2}}$ & -1 & 0 & $\delta_{2} \cdot e^{-0.5 i \varphi_{2}}$ & 0 & 0 \\
\hline 0 & 0 & $\delta_{1} \cdot e^{-0.5 i \varphi_{2}}$ & 0 & -1 & $\alpha_{2}$ & 0 & 0 \\
\hline 0 & 0 & 0 & 0 & $i G \cdot e^{-2 i \varphi_{3}}$ & -1 & 0 & $\sqrt{1-G^{2}} \cdot e^{-i \phi_{3}}$ \\
\hline 0 & 0 & 0 & 0 & $\sqrt{1-G^{2}} \cdot e^{-i \varphi_{3}}$ & 0 & -1 & iG \\
\hline 0 & $S_{12} \cdot e^{-2 i\left(\Phi_{1}+\varphi_{1}\right)}$ & 0 & 0 & 0 & 0 & $S_{22} \cdot e^{-2 i \varphi_{4}}$ & -1 \\
\hline
\end{tabular}

Where: $\varphi_{n}=2 \pi L_{n} / \lambda_{w g}$ - phase; $L_{n}$ - the length of waveguide sections; $f$-excitation frequency.

$M(f) \times\left(\begin{array}{c}a_{3}(f) \\ b_{3}(f) \\ a_{4}(f) \\ b_{4}(f) \\ a_{5}(f) \\ b_{5}(f) \\ a_{6}(f) \\ b_{6}(f)\end{array}\right)=\left(\begin{array}{c}0 \\ -a_{1} \cdot k_{1} \\ -a_{1} \cdot k_{1} \cdot e^{-0.5 i \varphi_{2}} \\ -a_{2} \cdot k_{2} \cdot e^{-0.5 i \varphi_{2}} \\ -a_{2} \cdot k_{2} \\ 0 \\ 0 \\ 0\end{array}\right)$ equations of the

Beam loading $-S_{12}(f)=S_{21}(f)=S_{12}(H F S S) \cdot \sqrt{1-\frac{\left(R_{s h} / Q\right) \cdot I_{\text {beam }}}{E_{\text {acc }}} \cdot \frac{2 \pi}{\beta_{g r}}}$

The transmission coefficient of the $S(f)$ - matrix of the accelerating structure is multiplied by the absorption coefficient of beam loading. $I_{\text {beam }}$ is the average pulse beam current, $E_{a c c}$ is the accelerating gradient, $\beta_{g r}$ the group velocity of the operation mode of accelerating structure.

After choosing input coupling, matcher reflection and relative phase and amplitudes of the input waves we can adjust the resonant ring, i.e. zero power reflection and zero backward wave into the TW section. We present here numerical simulation results for the resonance ring with the 15 cell accelerating structure ( $\sim 1 \mathrm{~m}$ length). The

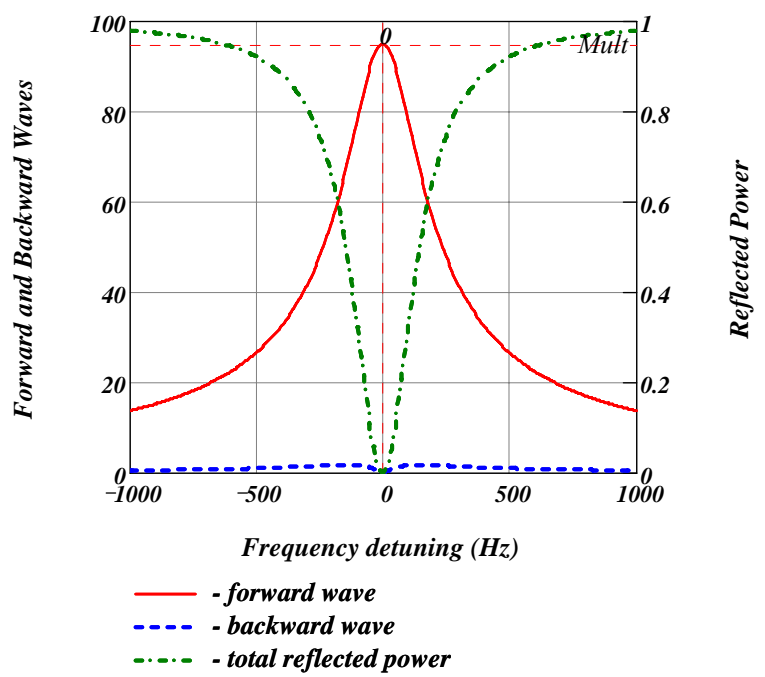

Figure 2. Amplitudes of forward wave, backward wave and total reflected power vs frequency detuning of the resonant ring. 
reflection coefficient is $-30.46 \mathrm{~dB}$ at $1300 \mathrm{MHz}, \varphi=105^{\circ}$, $R_{s h} / Q=1808 \Omega$, loaded with the beam current $I_{\text {beam }}=9 \mathrm{~mA}$ at the accelerating gradient $31.5 \mathrm{MeV} / \mathrm{m}$. Fig. 2 shows the well tuned resonance ring where the backward wave magnitude at the segments $L_{1}, L_{2}$ and $L_{3}$ is $\sim 10^{-4}$ of the forward wave.

If we suppose an acceptable level of backward wave into the section and reflected power from the resonant ring $1 \%$, the required tolerance of the ring parameters is presented in Tab. 2. As shown in the Table 2, the most precision and accuracy is needed for resonant ring frequency detuning. For a $1330 \mathrm{~mm}\left(4 \cdot \lambda_{w g}\right)$ waveguide loop length the acceptable error is $0.6 \mu \mathrm{m}$.

Table 2: Tolerance requirements of the two-coupler resonant ring parameters

\begin{tabular}{|l|l|}
\hline Parameter & Tolerance \\
\hline$\Delta L / L-$ feedback loop length & $4.5 \cdot 10^{-7}$ \\
\hline$\Delta L_{2} / L_{2}$ & $3.5 \cdot 10^{-2}$ \\
\hline$\Delta L_{4} / L_{4}$ & $2.4 \cdot 10^{-6}$ \\
\hline$\Delta k / k-$ inputs coupling & 0.0214 \\
\hline$\Delta \varphi-$ inputs phase difference & $\pm 1 \mathrm{deg}$ \\
\hline$\Delta G / G-$ matcher reflection & $4 \cdot 10^{-5}$ \\
\hline$\Delta Q_{\text {ext }} / Q_{\text {ext }}-$ loaded $Q$ factor & 0.13 \\
\hline$\Delta f_{0}-$ resonant frequency detuning & $\pm 15 \mathrm{~Hz}$ \\
\hline
\end{tabular}

It should be noted that with the proposed powering scheme there is no necessity for a high tuning frequency adjustment of the accelerating section itself at the chosen operational mode. The bandwidth of the coupling section of the structure and the additional phase advance due to the cavity frequency shift give a much smaller effect (by a few orders of magnitude) than the resonance ring frequency shift or the backward wave detuning. It is enough to control the overall resonant frequency and the backward wave suppression to achieve the standard operational parameters.

The second scheme uses only one non-directional input and special matcher, which splits the normal SW mode of resonant ring in two frequency shifted SW modes. These modes are excited with equal amplitudes and phase

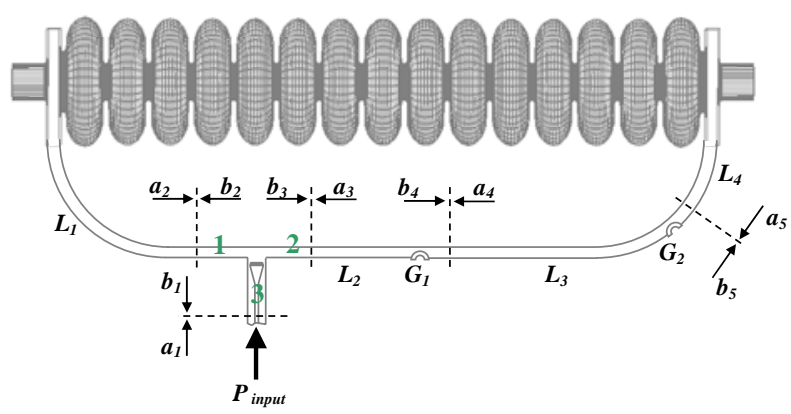

Figure 3. One-coupler model of the resonant travelling wave ring with STWA cavity. advance of $90^{\circ}$ with respect to each other. Their superposition is a travelling wave propagating along the ring. Our investigation it proved that it is better to use an additional matcher $G_{2}$ (see Fig. 3) to adjust independently the reflection from the TW section. Table 3 shows the similar results of one-coupler ring modelling to the previous two-coupler scheme.

Table 3: Tolerance requirements of the one-coupler resonant ring parameters

\begin{tabular}{|l|l|}
\hline Parameter & Tolerance \\
\hline$\Delta L / L-$ feedback loop length & $4.5 \cdot 10^{-7}$ \\
\hline$\Delta L_{2} / L_{2}$ & 0.01 \\
\hline$\Delta L_{3} / L_{3}$ & $4.1 \cdot 10^{-5}$ \\
\hline$\Delta L_{4} / L_{4}$ & $2.6 \cdot 10^{-5}$ \\
\hline$\Delta k / k-$ input coupling & 0.096 \\
\hline$\Delta G_{I} / G_{I}-$ first matcher reflection & $6 \cdot 10^{-5}$ \\
\hline$\Delta G_{2} / G_{2}-$ second matcher reflection & $1.07 \cdot 10^{-5}$ \\
\hline$\Delta Q_{\text {ext }} / Q_{\text {ext }}-$ loaded $Q$ factor & 0.13 \\
\hline$\Delta f_{0}-$ resonant frequency detuning & $\pm 15 \mathrm{~Hz}$ \\
\hline
\end{tabular}

\section{CONCLUSION}

The numerical model of the STWA structure with the feedback and input couplers is developed and tested. The model includes beam loading effects, and allows analysis of different schemes of structure excitation, tuning, tolerance requirements and beam loading. The two- or more coupler schemes can be exploited for excitation of a long SC TW section with high RF power consumption. The one-coupler scheme is beneficial for short $(\sim 1 \mathrm{~m})$ STWA, which can be used to replace ILC cavity.

\section{REFERENCES}

[1] V. Yakovlev, P. Avrakhov, et al. These proceedings, WEPMN066.

[2] International Linear Collider Reference Design Report, February 2007.

[3] R.B. R.-Shersby-Harvi and L.B. Mullett. Proc. Phys. SOC. London E, 270 (1949).

[4] G.A. Loew, R.B.Neal, in book: Linear Accelerators. Ed. P.Lapostole, E.Septier, North Holland Pub.

[5] R.B. Neal. Consideration of the use of Feedback in a Travelling Wave Superconducting Accelerator. Superconducting RF Cavities and Linacs, The 1968 Summer Study on Superconducting Devices and Accelerators at BNL, pp.111-126, 1968.

[6] Balakin V.E, Kazakov S.Yu, Solyak N.A, "RF deflection system of Gyrocon", Workshop on Relativistric RF electronics, Gorkii, 1982 (in Russian).

[7] M.M. Karliner, E.V. Kozyrev, I.G. Makarov, O.A. Nezhevenko, et al. Instrum. Methods Phys. Res., A 269, pp.459-473, 1988.

[8]. J.Altman. Microwave circuits, N.Y. 1964. 